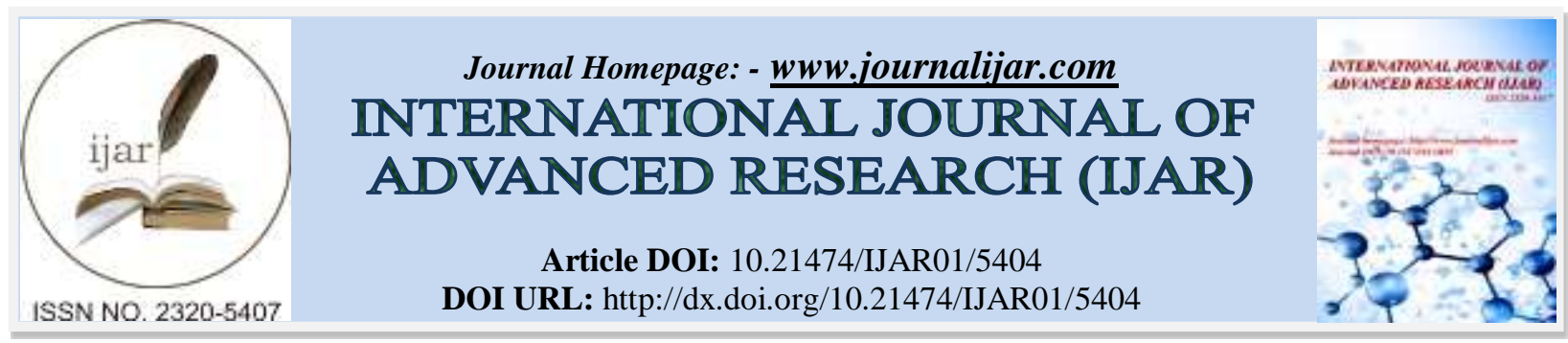

RESEARCH ARTICLE

\title{
NEUROLOGICAL AND OPHTHALMOLOGICAL RECOVERY AFTER LUMBO-PERITONEAL SHUNTING AS A TREATMENT OF IDIOPATHIC INTRACRANIAL HYPERTENSION
}

Moataz A. Elawady, MD and Mohamed M. Elmaghrabi, MD.

Lecturers of Neurosurgery Department, Faculty of Medicine, Benha University.

\section{Manuscript Info}

Manuscript History

Received: 12 July 2017

Final Accepted: 14 August 2017

Published: September 2017

Key words:-

Lumbo-peritoneal shunt, idiopathic intracranial hypertension.

\section{Abstract}

Background: Idiopathic intracranial hypertension (IIH) is a syndrome of unknown etiology resulting in increased intracranial pressure (ICP). Traditional options for medically refractory patients are CSF diversion or optic nerve sheath fenestration (ONSF).

Objective: to evaluate lumbo-peritoneal shunts in the treatment of idiopathic intracranial hypertension (IIH).

Patients and methods: A prospective clinical cohort study which including twenty two patients fulfilling the modified Dandy criteria for the diagnosis of IIH and were medically refractory underwent a lumbo-peritoneal shunt and were followed up for mean 26.5 \pm 3.23 months in Benha University hospital.

Results: headache improved in ten patients (45.5\%). Gradual resolution of papilloedema occurred with complete resolution of papilloedema in $4(18.2 \%), 14(63.6 \%)$ and $22(100 \%)$ patients at 2, 3 and 4 weeks postoperative respectively which is statistically significant.

Conclusion: Lumbo-peritoneal shunts are effective in treating idiopathic intracranial hypertension with transient and even mild complications.

Copy Right, IJAR, 2017,. All rights reserved.

\section{Introduction:-}

Idiopathic intracranial hypertension (IIH) is considered a syndrome of unknown etiology resulting in elevation in intracranial pressure (ICP) ${ }^{1,2}$ It is nearly having higher incidence in obese women of childbearing age. ${ }^{3}$

Both symptomatic and asymptomatic presentations are detected. The most common symptoms are headache, visual problems and tinnitus (pulsatile), but asymptomatic patients may be discovered in routine ophthalmological examination. ${ }^{4}$

Treatment modalities consist of conservative and surgical. Conservative treatment includes weight loss, drugs (commonly acetazolamide, furosemide and corticosteroids and less used by octreotide and topiramate) and serial lumbar punctures. Surgical procedures are CSF diversion (ventriculo-peritoneal and lumbo-peritoneal shunting), optic nerve sheath fenestration (ONSF), stenting of venous sinus and bariatric surgery. ${ }^{2}$

Actually no optimal management option for IIH has been approved. In large number of cases, medical option includes weight reduction often succeed in control the progression of $1 \mathrm{IH} .{ }^{5}$ However $25 \%$ of patients needs 
surgical/interventional procedures due to visual deficit or intractable headaches. ${ }^{6}$ Traditional options for a refractory patients to medical treatment are CSF shunting or ONSF. ${ }^{7}$ Recently used maneuvers are venous sinus stenting and bariatric surgery. ${ }^{2}$

We aim to evaluate the efficacy and complications of lumbo-peritoneal shunts in the treatment of IIH.

\section{Patients and Methods:-}

\section{Study design:-}

This is a prospective clinical study.

\section{Patients:-}

All patients fulfilling the modified Dandy criteria (table1) for diagnosing IIH and were refractory to medical and serial lumbar punctures treatment (developed either visual deficit progression or retractable headache) were included in this study and to each one a lumbo-peritoneal shunt was surgically inserted. This study was done in Benha University hospital from October 2013 to November 2016. This research accepted by Research Ethics Committee (REC) of faculty of medicine, Benha University. A written informed consent was taken from each patient after explaining all steps of this study.

Table 1:- Modified Dandy criteria ${ }^{8}$.

1. Symptoms of increased intracranial pressure as headache, nausea, vomiting, transient visual deficits or papilledema

2. False localizing sign: abducent (CN VI) palsy

3. The patient is fully conscious and alert

4. Negative CT and MRI findings Without evidence suggesting cerebral thrombosis

5. Opening pressure of lumbar puncture is $>25 \mathrm{~cm} \mathrm{H} 2 \mathrm{O}$ With normal CSF biochemistry and cytological analysis

6. No other cause for increased intracranial pressure

Adapted from Friedman and Jacobson ${ }^{9}$

\section{Preoperative workup:-}

Initially complete meticulous neurological examination and neurological follow up was done every week.

The evaluation of headache was determined using the visual analogue score (VAS) for pain and this score was checked and recorded for every patient daily. Reviewing of the radiographic images, CT and MRI brain, in all patients was done by expert radiologist.

The ophthalmological examinations were done, by the same expert ophthalmological consultant, including the visual acuity, fundus examination and visual field (figure 1). Papilledema grading using the Frisén scale grade is applied. ${ }^{10}$

\section{Surgical note:-}

LPS was done under general anesthesia after a percutaneous procedure by a spinal 14-gauge needle. Laminectomy was not needed in any patient. Eight to ten centimeters length of the lumbar proximal end of the shunt system was introduced at lumbar 2-3 or 3-4 levels and was directed to move cranially. In all included patients a medium pressure valve was used. Antisiphon valve shunt was not used due its cost. A broad spectrum antibiotic for about 7 days was used postoperatively in all patients.

\section{Postoperative follow up:-}

All patients were examined neurologically and ophthalmologically in early postoperative period and weekly for the first month then became monthly for the first year and then follow up every three months in second year (mean $26.5 \pm 3.23$ months). Radiological evaluation was done at early postoperative period and $6^{\text {th }} \& 12^{\text {th }}$ month \pm after 2 years using CT or MRI brain, 3D CT dorsolumbar spine and pelvi-abdominal sonography by the same radiologist.

\section{Statistical analysis:-}

Software (SPSS, Version 20.0 for Windows, SPSS Inc, Chicago, IL) was used for analyzes of the data. Qualitative variables were summarized as frequency and percentages while quantitative data as mean $\pm \mathrm{SD}$. Mc Nemar test was applied for the comparison of quantitative variables before, after operation and follow up periods. A P value $<0.05$ was considered statistically significant $(*)$ while $>0.05$ statistically insignificant. 


\section{Results:-}

Patient details:-

Twenty two patients fulfilling the modified Dandy criteria (table1) for the diagnosis of IIH and were medically refractory (visual deterioration in 14 patients and uncontrollable headache in all patients) were included in this study. They were 20 females and 2 males with age ranging from 22 to 55 years (Mean \pm SD: $38.7 \pm 8.3$ ) with the mean height $160.3 \mathrm{~cm}$ and mean weight $92.6 \mathrm{~kg}$ and mean body mass index (BMI) 36.1. Table 2 shows our patient criteria.

Table 2:- Patient Criteria

\begin{tabular}{|l|l|}
\hline Patient criteria & Value $(\mathbf{n = 2 2})$ \\
\hline Age /years mean \pm SD (range) & $38.7 \pm 8.3(22-55)$ \\
Sex & $2(9.1)$ \\
$\quad$ Male & $20(90.9)$ \\
$\quad$ Female & \\
Complaint & $22(100)$ \\
Uncontrollable headache & $14(63.6)$ \\
Reduced vision & $6(27.3)$ \\
Nausea and/ or vomiting & \\
Grade of papilloedema & $14(63.6)$ \\
$\quad$ III & $8(36.4)$ \\
\hline IV & \\
\hline
\end{tabular}

\section{Clinical results:-}

Headache improved in ten patients $(45.5 \%)$. Gradual resolution of papilloedema occurred which is statistically significant as shown in table 3; complete resolution of papilloedema in 4(18.2\%), 14(63.6\%) and 22(100\%) patients at 2, 3 and 4 weeks postoperative respectively.

Table 3:- improvement of papilloedema grades in studied group.

\begin{tabular}{|l|l|l|l|l|}
\hline & Preop & 2 weeks postop & 3 weeks postop & 4 weeks postop \\
\hline No papilloedema & $0(0.0)$ & $4(18.2)$ & $14(63.6)$ & $22(100)$ \\
\hline Grade I papilloedema & $0(0.0)$ & $8(36.4)$ & $6(27.3)$ & $0(0.0)$ \\
\hline Grade II papilloedema & $0(0.0)$ & $6(27.3)$ & $2(9.1)$ & $0(0.0)$ \\
\hline Grade III papilloedema & $14(63.6)$ & $4(18.2)$ & $0(0.0)$ & $0(0.0)$ \\
\hline Grade IV papilloedema & $8(36.4)$ & $0(0.0)$ & $0(0.0)$ & $0(0.0)$ \\
\hline $\mathrm{P}=0.001^{* *}$ (using Mc Nemar test) \\
\hline
\end{tabular}




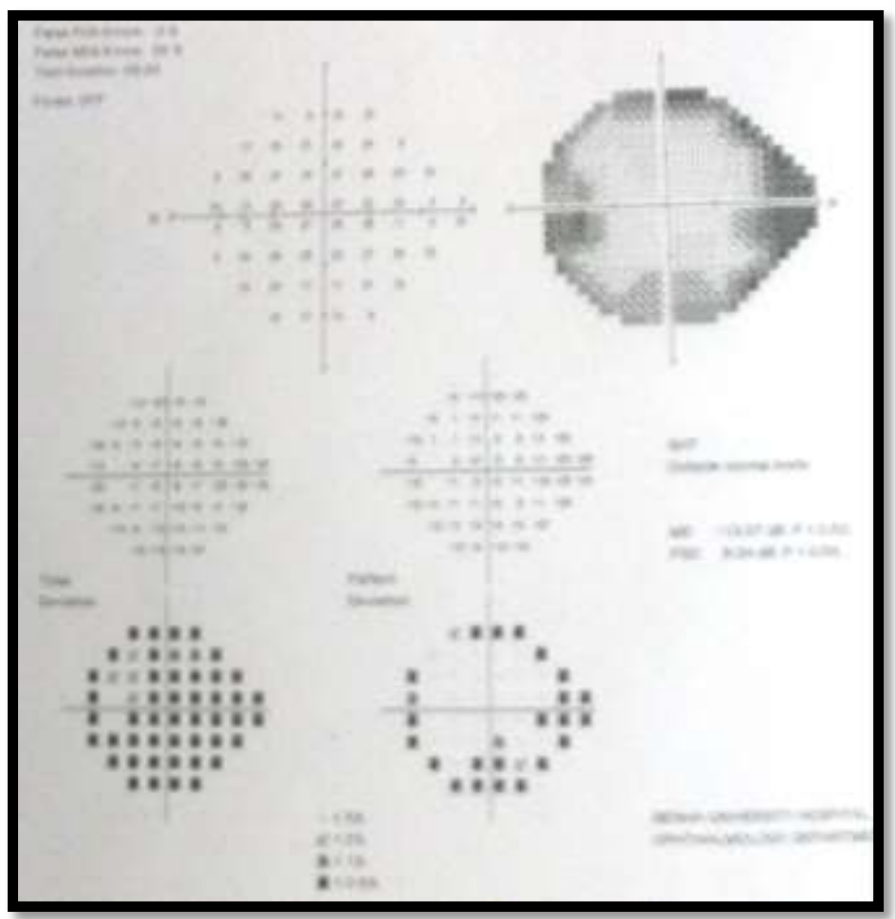

Fig.1:- Pre-operative Visual field of the left eye performed by Humphery automated perimetry with acceptable reliability indices showing severe generalized reduction of retinal sensitivity, and central scotoma together with advanced annular defect giving rise to tubular vision.

\section{Complications:-}

Twelve patients developed complications (Table 4) during the follow-up period. No shunt-related deaths occurred.

Eight patients developed temporary over drainage in the form of headache on sitting and walking only, while there was no headache in resting stage; received intravenous fluid therapy and maintained in recumbent position; symptoms were improved gradually.

An obese two females had recurrent preoperative visual symptoms with aggravated headache and a secondary CT examination detected that disconnection of the distal tube was observed. Revision surgery to reconnect the distal tube was successful after 28 months follow up as in figures $2 \& 3$. Another two patients, a male and a female, presented with CSF leak from the back wound and recurrence of symptoms, 3D-CT imaging showed slipped peritoneal end of the LPS with coiling of the tube as in figure 4. Surgical exploration was conducted and suspected extra-peritoneal insertion of peritoneal tube in the two patients so it was re-inserted after confirming its integrity.

Table 4:- List of complications

\begin{tabular}{|c|c|c|}
\hline Comp & & Number of patients $=22(\%)$ \\
\hline$\bullet$ & Overdrainage & $8(36.4)$ \\
\hline $\begin{array}{l}\bullet \\
\text { tube) }\end{array}$ & CSF leak (Extra-peritoneal insertion of abdominal & $2(9.1)$ \\
\hline$\bullet$ & Disconnection of distal tube & $2(9.1)$ \\
\hline
\end{tabular}




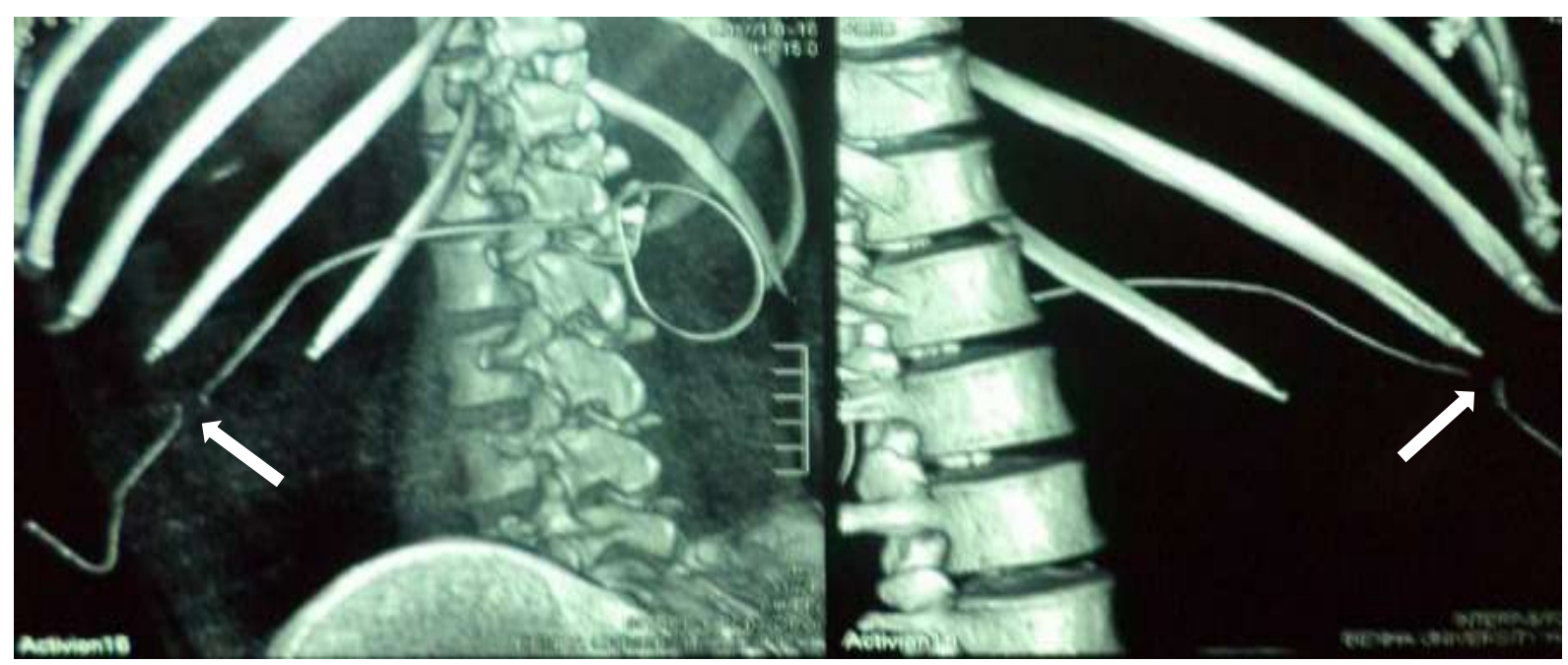

Fig. 2:- Showing a case with disconnected distal tube (arrow)

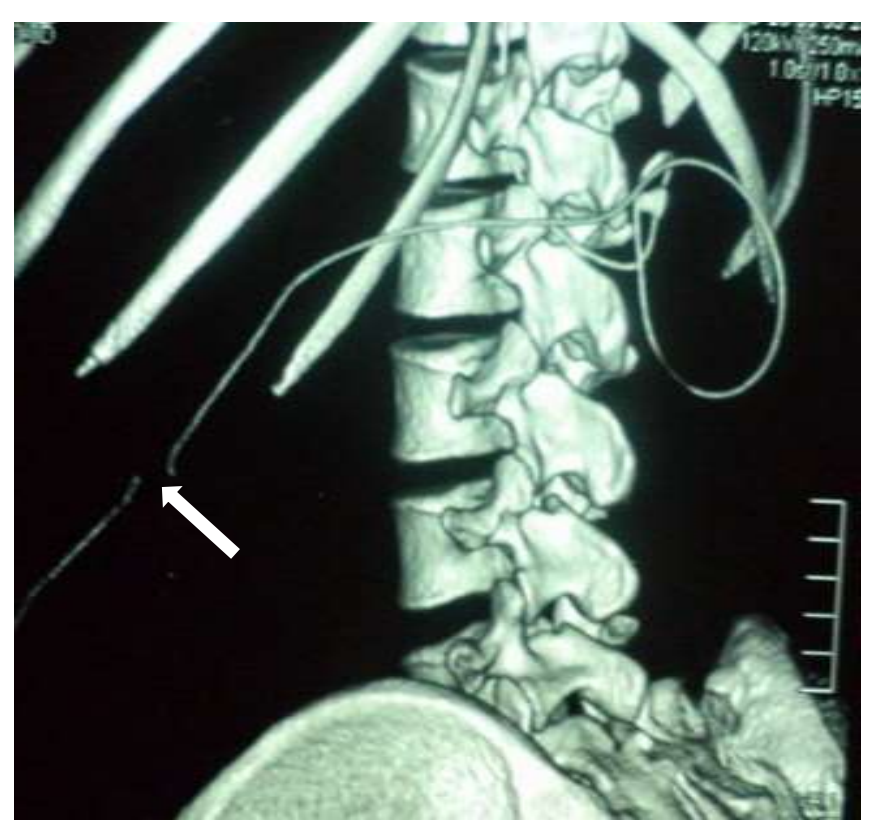

Fig. 3:- Showing tube disconnection (arrow) 


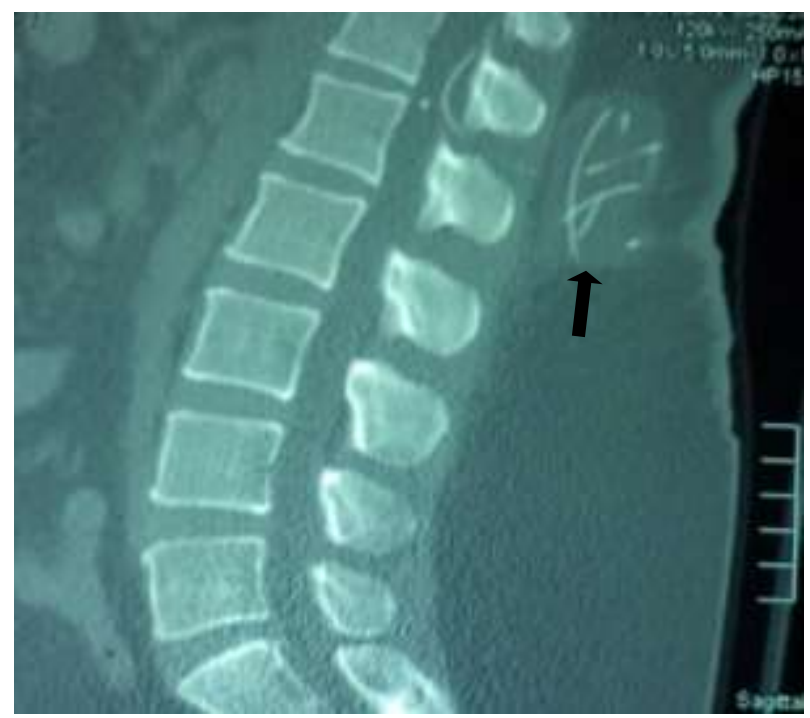

Fig. 4:- CT imaging showing a case had slipped peritoneal end of the LPS with coiling of the tube (arrow)

\section{Discussion:-}

Lumbo-peritoneal and ventriculo-peritoneal shunts are still a frequent treatment option for idiopathic intracranial hypertension. Although these types of shunts have been used in institutes for a long time, it is actually not clear how the total CSF volume is changed and what are more affected portions of cranial and spinal fluid? ${ }^{11}$ The lumboperitoneal shunts (LPS) were suggested that decreasing the spinal fluid volume, while the ventriculo-peritoneal shunts (VPS) keep both the cranial and spinal fluid volumes in accepted physiological range. In previous literatures, normal cranial and spinal fluid volumes were a prognostic factor for a good clinical outcome results in IIH. ${ }^{12}$

In our study, LPS only was inserted in all patients due to very small ventricles.

Being an extra-cranial procedure, LPS insertion has a lower complication rate. Shunt obstruction and infection are the most common complications of this operation. ${ }^{11}$ The complications that observed in our patients although occurred in more than half of the patients were mostly mild and transient. A CT based study by found a $70 \%$ incidence of acquired type I Chiari malformation after a LPS, with reported associated syrinx in about $50-70 \%{ }^{8}$ No syrinx formation was detected in our patients by MRI postoperative findings.

In supported literatures of the favorable outcome of LPS procedure for treatment of IIH found that compared various surgical techniques for management of visual deficit in IIH refractory to medical treatment and reported significant improvement or complete recovery of visual deficit in $38.7 \%$ of patients after VPS procedure and $47 \%$ after stenting, but in $44.6 \%$ of patients after LPS procedure and $80 \%$ after optic ONSF rather than documented rare visual worsening for all previous procedures. ${ }^{13}$

In our study, the twenty two patients improved gradually to be fundus free of all at four weeks post operatively. A retrospectively reviewed clinical records of eighteen patients with LPS insertion found that fourteen patients (77.8\%) with preoperative headache did not complain of headache postoperatively, and four $(22.2 \%)$ had headache not related to shunt function, two of the patients $(11.1 \%)$ with preoperative visual complain which visual problems were reported postoperatively and thirteen patients $(72.2 \%)$ did not require shunt revision so considered that LPS is effective in treatment of IIH. ${ }^{14}$ But in our patients, eight cases $(36.4 \%)$ of post-operative headache due to intracranial hypotension which improved gradually under conservative treatment.

In comparison between the outcome of IIH treatment by VPS and LPS, both shunts were found to be effective in controlling the clinical manifestations of IIH in early postoperative period with no statistically significant higher VPS failure rate and LPS revision rate. ${ }^{3}$ Another study of twenty two patients, with failure or complications of medical treatment and underwent LPS for IIH, reported that recovery of headache in 19(86.4\%) patients and complete resolution of papilledema in $16(72.7 \%$ ) patients and concluded that LPS is easy with effective maneuver to interfere with intractable headache and visual impairment produced by IIH. $^{15}$ 
In our study, only four patients (18.2\%) needed surgical revision due to disconnection or slipped peritoneal end (equally $9.1 \%$ of each).

\section{Conclusion:-}

Lumbo-peritoneal shunts are effective in treating idiopathic intracranial hypertension with transient and even mild complications.

\section{References:-}

1. Biousse V, Bruce BB, Newman NJ: Update on the pathophysiology and management of idiopathic intracranial hypertension. J Neurol Neurosurg Psychiatry; 83:488-94, 2012.

2. Kalyvas AV, Hughes M, Koutsarnakis C, Moris D, Liakos F, Sakas DE, Stranjalis G: Efficacy, complications and cost of surgical interventions for idiopathic intracranial hypertension: a systematic review of the literature. Acta Neurochir; 159:33-49, 2017.

3. Abubaker K, Ali Z, Raza K, Bolger C, Rawluk D, O'Brien D: Idiopathic intracranial hypertension: lumboperitoneal shunts versus ventriculoperitoneal shunts—case series and literature review. Br J Neurosurg; 25:94-99, 2011.

4. Galvin JA, Van Stavern GP: Clinical characterization of idiopathic intracranial hypertension at the Detroit Medical Center. J Neurol Sci; 223:157-160, 2004.

5. Brazis PW: Clinical review: the surgical treatment of idiopathic pseudotumour cerebri (idiopathic intracranial hypertension). Cephalalgia; 28:1361-1373, 2008.

6. Corbett JJ, Thompson HS: The rational management of idiopathic intracranial hypertension. Arch Neurol; 46:1049-1051, 1989.

7. Galgano MA, Deshaies EM: An update on the management of pseudotumorcerebri. Clin Neurol Neurosurg; 115:252-259, 2013.

8. Chumas PD, Armstrong DC, Drake JM, Hoffman HJ, Humphreys RP, Rutka JT.: Tonsillar herniation: the rule rather than the exception after lumboperitoneal shunting in the pediatric population. J Neurosurg; 78:568-573, 1993.

9. Friedman DI, Jacobson DM: Diagnostic criteria for idiopathic intracranial hypertension. Neurology; 59:1492$1495,2002$.

10. Frisén L: Swelling of the optic nerve head: a staging scheme. J Neurol Neurosurg Psychiatry; 45:13-18, 1982.

11. Gupta B, Panigrahi M: Acquired Chiari I malformation following a lumbo-peritoneal shunt- William's hypothesis revisited. J Med Sci Res; 1(1):13-15, 2013.

12. Nikić I, Radoš M, Frobe A et al: Effects of lumboperitoneal and ventriculoperitoneal shunts on CSF. Croat Med J; 57:293-7, 2016.

13. Feldon SE: Visual outcomes comparing surgical techniques for management of severe idiopathic intracranial hypertension. Neurosurg Focus; 235:E6, 2007.

14. Toma AK, Dherijha M, Kitchen ND, Watkins LD: Use of lumboperitoneal shunts with the Strata NSC valve: a single-center experience. J Neurosurg; 1136:1304-8, 2010.

15. El-Saadany WF, Farhoud A, Zidan I: Lumboperitoneal shunt for idiopathic intracranial hypertension: patients' selection and outcome. Neurosurg Rev; 35:239-444, 2012. 